\title{
PARTITIONS WITH CONGRUENCE CONDITIONS
}

\author{
M. M. ROBERTSON
}

\begin{abstract}
Let $A=\cup_{i=1}^{q}\{a(i)+\nu M: \nu=0,1,2, \ldots\}$, where $q, M$ and the $a(i)$ are positive integers such that $a(1)<a(2)<\cdots<a(q) \leqslant M$. Asymptotic formulae are obtained for $p(n, k, A), p^{*}(n, k, A)$ the number of partitions of $n$ into $k$ parts, $k$ unequal parts respectively, where all the parts belong to $A$.
\end{abstract}

Since the celebrated Hardy-Ramanujan paper [4] which gave an asymptotic formula for $p(n)$, the number of unrestricted partitions of the positive integer $n$, many authors have evaluated different cases of $p(n, A)$, the number of partitions of $n$ into parts belonging to

$$
A=\bigcup_{i=1}^{q}\{a(i)+\nu M: \nu=0,1,2, \ldots\},
$$

where $q, M$ and the $a(i)$ are positive integers such that $a(1)<a(2)<\ldots$ $<a(q) \leqslant M$. The Hardy-Ramanujan circle method as modified by Rademacher [9] was employed to obtain convergent series expressions for $p(n, A)$. When $q=2, a(2)=M-a(1)$, Niven [8] investigated the case $M=6$, Lehner [6] the case $M=5$ and Livingood [7] the case where $M$ is any prime $>3$. Later Iseki [5] evaluated $p(n, A)$ when $M$ is any composite $>3$ and $a(1), M$ are relatively prime. Then Hagis [3] considered the case of all odd primes $M$, where $q=2 r$ and $a(h)+a(2 r-h)=M$ for $1 \leqslant h \leqslant r$.

In all these cases, $A$ is symmetric in the sense that $a(h) \in A$ implies that $M-a(h) \in A$ and this ensures that the generating function of the $p(n, A)$ is a modular form. Rademacher's method then leads to a convergent series representation of $p(n, A)$. Grosswald [2] investigated the case where $M$ is any odd prime and $A$ is an arbitrary asymmetrical set. Then the above method cannot be applied and only asymptotic results are obtained.

In [1], Erdös and Lehner by means of more elementary methods investigated $p(n, k)$, the number of partitions of $n$ into $k$ parts, and proved that $p(n, k) \sim n^{k-1} / k !(k-1) !$ as $n \rightarrow \infty$, provided that $k=o\left(n^{1 / 3}\right)$. This formula does not appear to have been generalized in the above manner and it is the object of this note to do so.

We denote by $p(n, k, A), p^{*}(n, k, A)$ the number of partitions of $n$ into $k$ parts, $k$ unequal parts respectively, where all the parts belong to $A$. For every

Received by the editors September 29, 1975.

AMS (MOS) subject classifications (1970). Primary 10J20, 10A45; Secondary 10A10.

Key words and phrases. Partition, asymptotic, congruence. 
positive integer $n$, we define $r$ by $r \equiv n(\bmod M), 1 \leqslant r \leqslant n$. We write

$$
\begin{aligned}
P(x, k, A) & =\left\{\sum_{i=1}^{q} x^{a(i)}\right\}^{k}=\sum_{\nu=1}^{k M} c_{\nu} x^{\nu}, \\
S_{r}\{P(x, k, A)\} & =\sum_{\nu=0}^{k-1} c_{\nu M+r}
\end{aligned}
$$

and $d, \delta$ for the greatest common divisors $(a(1), \ldots, a(q), M),(a(2)$ $-a(1), \ldots, a(q)-a(1), M) .\{\delta=M$ for $q=1$. $\}$ Clearly $d=(a(1), \delta)$. We can now state our result.

THEOREM. For any given $k, r$ for which $S_{r}\{P(x, k, A)\} \neq 0$,

$$
p(M n+r, k, A) \sim p^{*}(M n+r, k, A) \sim n^{k-1} S_{r}\{P(x, k, A)\} / k !(k-1) !
$$

as $n \rightarrow \infty$. If $\delta$ divides $r, n \rightarrow \infty$ and $k \rightarrow \infty$ through multiples of $\delta / d$ subject to the condition that $k=o\left(n^{1 / 4}\right)$, then

$$
p(M n+r, k, A) \sim p^{*}(M n+r, k, A) \sim n^{k-1} q^{k} \delta / M\{k !(k-1) !\} .
$$

We observe that the second part of our theorem is established only for $k=o\left(n^{1 / 4}\right)$ instead of under the condition $k=o\left(n^{1 / 3}\right)$ of Erdös and Lehner. The proof here is, in fact, quite different from that of [1] where the condition on $k$ is obtained by using the fact that the number of partitions of $n$ into $k$ unequal parts is $p\left(n-\frac{1}{2} k(k-1), k\right)$. This appears to have no obvious generalization for partitions into parts belonging to $A$.

Proof of THe Theorem. We write

$$
\begin{aligned}
G(y) & =G(y, x, A)=\prod_{\nu=0}^{\infty} \prod_{i=1}^{q}\left\{1-x^{\nu M+a(i)} y\right\}^{-1} \\
& =1+\sum_{k=1}^{\infty} g(k) y^{k},
\end{aligned}
$$

where $g(k)=g(k, x, A)$ is the generating function of $p(n, k, A)$. Therefore,

$$
\begin{aligned}
\log G(y) & =-\sum_{\nu=0}^{\infty} \sum_{i=1}^{q} \log \left\{1-x^{\nu M+a(i)} y\right\} \\
& =\sum_{\nu=0}^{\infty} \sum_{i=1}^{q} \sum_{h=1}^{\infty} h^{-1} x^{h\{\nu M+a(i)\}} y^{h} \\
& =\sum_{h=1}^{\infty} h^{-1} y^{h} \beta(h),
\end{aligned}
$$

where

$$
\beta(h)=\beta(h, x, A)=\left(1-x^{h M}\right)^{-1} \sum_{i=1}^{q} x^{h a(i)} .
$$

Hence 


$$
G(y)=\exp \left\{\sum_{h=1}^{\infty} h^{-1} y^{h} \beta(h)\right\}
$$

and so,

$$
g(k)=\sum_{(k)} \prod_{m}\{h(m) !\}^{-1}\left\{m^{-1} \beta(m)\right\}^{h(m)},
$$

where the sum is taken over all unrestricted partitions of $k$ of the form $k=\sum_{m=1}^{k} h(m) m$ and the product is taken over all the different parts $m$ of the partition.

The term on the right-hand side of (4) corresponding to the partition of $k$ into $k$ units is

$$
\begin{aligned}
(k !)^{-1}\left(1-x^{M}\right)^{-k} & \left\{\sum_{i=1}^{q} x^{a(i)}\right\}^{k} \\
& =(k !)^{-1}\left(1-x^{M}\right)^{-k} \sum_{\nu=1}^{k M} c_{\nu} x^{\nu}
\end{aligned}
$$

by (1), and the coefficient of $x^{M n+r}$ in this term is

$$
(k !)^{-1} \sum_{\nu=0}^{k-1} c_{\nu M+r}\left(\begin{array}{c}
n-\nu+k-1 \\
k-1
\end{array}\right) \text {. }
$$

It follows that, as $n \rightarrow \infty$, this coefficient

$$
\begin{aligned}
& \sim n^{k-1} \sum_{\nu=0}^{k-1} c_{\nu M+r} / k !(k-1) ! \\
& =n^{k-1} S_{r}\{P(x, k, A)\} / k !(k-1) !
\end{aligned}
$$

by (2).

From (3), the general term on the right-hand side of (4) is

$$
\prod_{m}\{h(m) !\}^{-1} m^{-h(m)}\left(1-x^{m M}\right)^{-h(m)}\left\{\sum_{i=1}^{q} x^{m a(i)}\right\}^{h(m)} .
$$

Since, for all $\nu>0$, the coefficient of $x^{\nu}$ in $\sum_{i=1}^{q} x^{m a(i)}$ is not greater than the coefficient of $x^{\nu}$ in $\left\{\sum_{i=1}^{q} x^{a(i)}\right\}^{m}$, the coefficient of $x^{M n+r}$ in this general term is less than

$$
C n^{-1} \prod_{m} n m^{-h(m)}(n / m)^{h(m)-1} S_{r}\{P(k, A)\} / h(m) !\{h(m)-1\} !,
$$

where $C$ is independent of $n, k$. Hence, in order to show that

$$
p(M n+r, k, A) \sim n^{k-1} S_{r}\{P(x, k, A)\} / k !(k-1) !
$$

as $n \rightarrow \infty$, we must prove that

$$
n^{-k} k !(k-1) ! \sum_{(k)}^{\prime} \prod_{m} n^{h(m)} m^{1-2 h(m)} / h(m) !\{h(m)-1\} !=o(1)
$$


as $n \rightarrow \infty$, where the sum is taken over all partitions $\sum h(m) m$ of $k$ into less than $k$ parts.

Now, since any partition of $k$ into $k-\mu$ parts, where $\mu<\frac{1}{2} k$, must contain at least $k-2 \mu$ units, we have

$$
\prod_{m} h(m) !\{h(m)-1\} ! \geqslant \Lambda(k-2 \mu)
$$

where $\Lambda(k-2 \mu)=(k-2 \mu) !(k-2 \mu-1)$ ! for $\mu<\frac{1}{2} k$ and $\Lambda(k-2 \mu)=1$ for $\mu \geqslant \frac{1}{2} k$. Also, the number of partitions of $k$ into $k-\mu$ parts is less than or equal to $p(\mu)$, the number of unrestricted partitions of $\mu$, according as $\mu>\frac{1}{2} k$ or $\mu \leqslant \frac{1}{2} k$. Therefore, since for all $\mu>0, p(\mu)<\exp \{\pi \sqrt{ }(2 \mu / 3)\}$, the left-hand side of (6) is less than

$$
\begin{aligned}
& \sum_{\mu=1}^{k-1} \exp \{\pi \sqrt{ }(2 \mu / 3)\} k^{4 \mu} n^{-\mu} \\
&<\sum_{\mu=1}^{k-1} \exp \{\pi \sqrt{ }(2 \mu / 3)+4 \mu \log k-\mu \log n\} \\
&<C^{\prime} \sum_{\mu=1}^{\infty} \exp \left\{-\frac{1}{2} \mu \log \left(n / k^{4}\right)\right\},
\end{aligned}
$$

where $C^{\prime}$ is independent of $n, k$. (6) follows immediately.

If we write

$$
\begin{aligned}
G^{*}(y) & =G^{*}(y, x, A)=\prod_{\nu=0}^{\infty} \prod_{i=1}^{q}\left\{1+x^{\nu M+a(i)} y\right\} \\
& =1+\sum_{k=1}^{\infty} g^{*}(k) y^{k},
\end{aligned}
$$

then it follows that

$$
\log G^{*}(y)=\sum_{h=1}^{\infty}(-1)^{h-1} h^{-1} y^{h} \beta(h)
$$

and

$$
g^{*}(k)=\sum_{(k)} \prod_{m}\{h(m) !\}^{-1}\left\{(-1)^{m-1} m^{-1} \beta(m)\right\}^{h(m)} .
$$

The proof of the asymptotic formula for $p^{*}(M n+r, k, A)$ then follows from (7) exactly as the proof of the formula for $p(M n+r, k, A)$ followed from (4).

In order to prove the second part of the theorem, we let $\omega$ be a primitive $M$ th root of unity. Then, from (1) and (2),

$$
P\left(\omega^{h}, k, A\right)=\sum_{\nu=1}^{k M} c_{\nu} \omega^{\nu h}=\sum_{r=1}^{M} S_{r} \omega^{r h}
$$

for $1 \leqslant h \leqslant M$, where $S_{r}=S_{r}\{P(x, k, A)\}$. It is a simple application of alternant theory to solve these equations and obtain 


$$
S_{r}=M^{-1} \sum_{\nu=0}^{M-1} \omega^{-r \nu} P\left(\omega^{\nu}, k, A\right)
$$

for $1 \leqslant r \leqslant M$. From the definition of $\delta, \sum_{i=1}^{q} \omega^{\nu a(i)}=q \omega^{\nu a(1)}$ whenever $M / \delta$ divides $\nu$ and $\left|\sum_{i=1}^{q} \omega^{\nu a(i)}\right|<q$ otherwise. Hence, since $\delta$ divides $r$ and $(a(1), \delta)=d$, we see from (1) and (8) that $S_{r} \sim q^{k} \delta / M$ as $k \rightarrow \infty$ through multiples of $\delta / d$. The second part of the theorem now follows from (5) by letting $n, k \rightarrow \infty$, since (6) follows as before for $k=o\left(n^{1 / 4}\right)$.

\section{REFERENCES}

1. P. Erdös and J. Lehner, The distribution of the number of summands in the partitions of $a$ positive integer, Duke Math. J. 8 (1941), 335-345. MR 3, 69.

2. E. Grosswald, Some theorems concerning partitions, Trans. Amer. Math. Soc. 89 (1958), 113-128. MR 20 \#3840.

3. P. Hagis, $A$ problem on partitions with a prime modulus $p \geqslant 3$, Trans. Amer. Math. Soc. 102 (1962), 30-62. MR 26 \#3688.

4. G. H. Hardy and S. Ramanujan, Asymptotic formulae in combinatory analysis, Proc. London Math. Soc. (2) 17 (1918), 75-115.

5. S. Iseki, A partition function with some congruence condition, Amer. J. Math. 81 (1959), 939-961. MR 21 \# 7189.

6. J. Lehner, A partition function connected with the modulus five, Duke Math. J. 8 (1941), 631-655. MR 3, 166 .

7. J. Livingood, A partition function with the prime modulus $p>3$, Amer. J. Math. 67 (1945), 194-208. MR 6, 259.

8. I. Niven, On a certain partition function, Amer. J. Math. 62 (1940), 353-364. MR 1, 201.

9. H. Rademacher, On the partition function p(n), Proc. London Math. Soc. (2) 43 (1937), 241-254.

Department of Mathematics, University of SURrey, Guildford, SURRey, England 